\title{
The Perfect Storm: A Case of Non-Uremic Calciphylaxis
}

\author{
Manuel Martins Barbosa, Elsa Araújo, Marta Matos Pereira, Tiago Mendes \\ Unidade Local Saúde Alto Minho, EPE, Ponte de Lima, Portugal
}

Doi: 10.12890/2019_001078 - European Journal of Case Reports in Internal Medicine - @ EFIM 2019

Received: 03/03/2019

Accepted: $11 / 03 / 2019$

Published: $17 / 04 / 2019$

How to cite this article: Martins Barbosa M, Araújo E, Matos Pereira M, Mendes T. The perfect storm: a case of non-uremic calciphylaxis. EJCRIM 2019;6: doi:10.12890/2019_001078.

Conflicts of Interests: The Authors declare that there are no competing interests.

This article is licensed under a Commons Attribution Non-Commercial 4.0 License

\section{ABSTRACT}

Calciphylaxis is a severe condition associated with chronic kidney disease. We describe a rare case of non-uremic calciphylaxis in a 79-yearold woman with prosthetic aortic and mitral valves, chronic kidney disease and anaemia, who presented with painful skin lesions on her left leg. She was taking warfarin, calcium and iron supplements, and had been recently diagnosed with oligoarthritis and started on prednisolone, all factors associated with calciphylaxis. Despite treatment, the lesions rapidly progressed to large ulceration and sepsis, and pain management was challenging. Calciphylaxis was confirmed following a second skin biopsy but the patient died from septic shock and multi-organ failure. This case highlights the challenges associated with the diagnosis and treatment of calciphylaxis despite a high level of suspicion.

\section{LEARNING POINTS}

- The number of reported cases of calciphylaxis, once considered very rare, is increasing.

- Physicians should be aware of new information concerning the condition.

- Treatment includes a low antibiotic threshold, comprehensive pain management and efficient wound care.

\section{KEYWORDS}

Non-uremic calciphylaxis, calciphylaxis lesions, wound care, iatrogenesis, CKD complications

\section{CASE DESCRIPTION}

A 79-year-old woman was admitted to the internal medicine ward with painful lesions on her lower left leg of recent onset. Her medical history included prosthetic aortic and mitral valves, chronic ischaemic stage 4 kidney disease, multifactorial anaemia (due to valvar leak, gastrointestinal loss and chronic kidney disease) and a recent diagnosis of oligoarthritis of unknown aetiology. She was under the care of cardiology, internal medicine and rheumatology clinicians.

The patient had been on warfarin for over a decade. She was also taking calcium carbonate supplements, receiving weekly intravenous iron supplements, and had been recently started on prednisolone $20 \mathrm{mg}$ daily for the oligoarthritis.

The lesions were described as initially small violaceous nodules with diameter of $3 \mathrm{~cm}$ or less on the posterior side of the patient's left leg. By the time of admission, they had progressed to typical stellate ulcers (Fig. 1). The initial differential diagnosis by a dermatologist was warfarinrelated ulcers, vascular ulcers or calciphylaxis. Warfarin was suspended and replaced with low molecular weight heparin adjusted for renal function. A first skin biopsy was inconclusive.

Within 5 days the small nodules had developed into necrotic ulcers with eschars that covered the entire left calf. General surgery, palliative care and nutrition specialists were consulted. Pain control and wound management were essential as the necrotic bed was spreading and there was a risk of superimposed infection (Figs. 2 and 3). Pain management was also challenging as the patient reacted with confusion and agitation to an opioid combination. Administration of a morphine bolus, transdermal fentanyl and oral gabapentin was effective. 


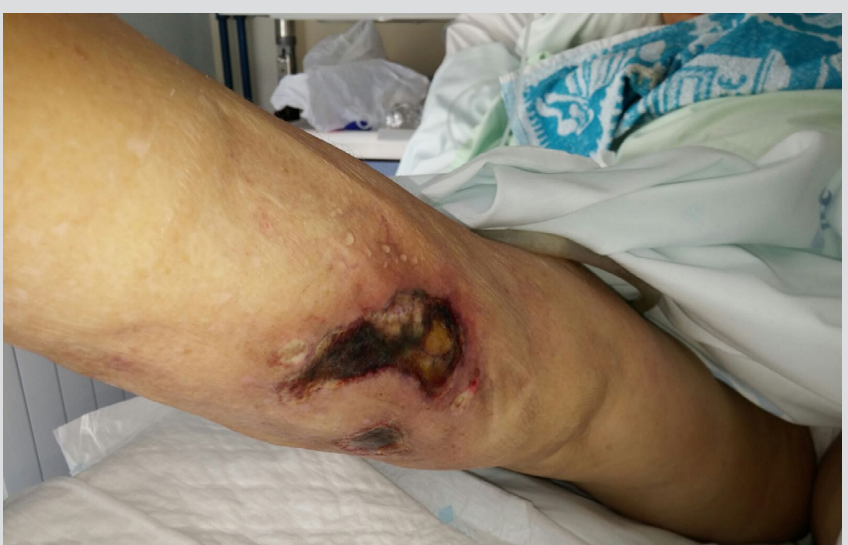

Figure 1. Lesions on admission

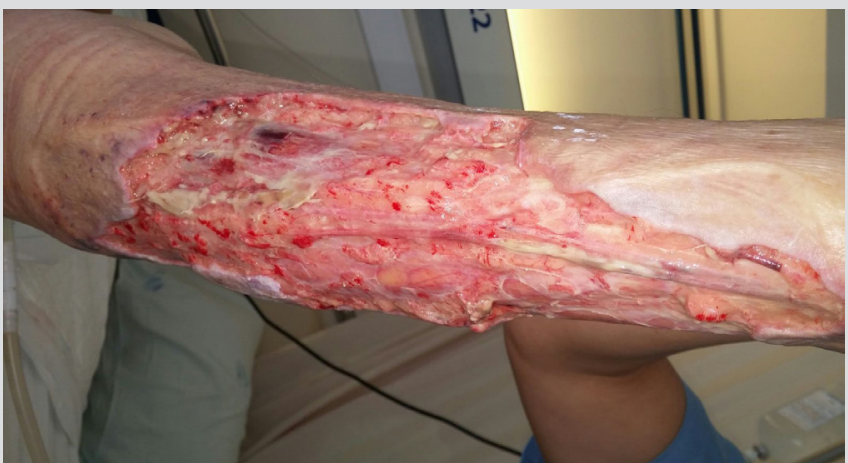

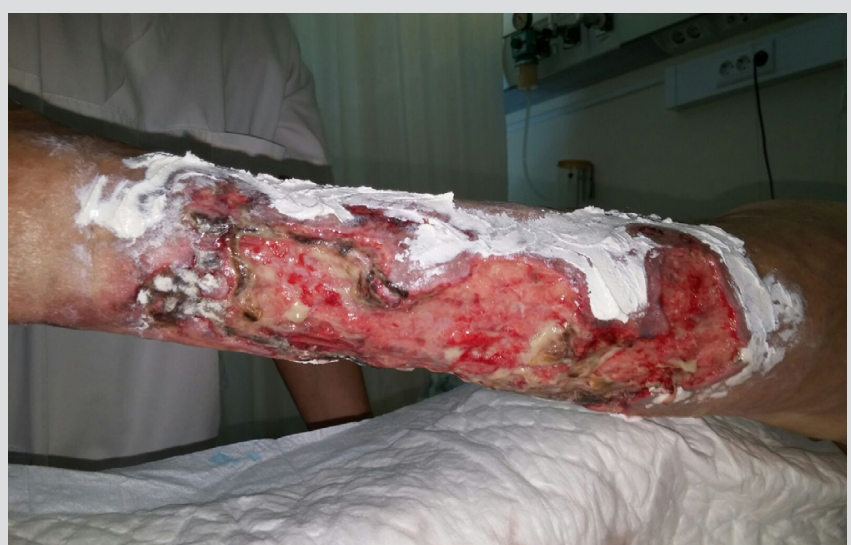

Figure 2. Lesions evolving to a large ulcer, 7 days after admission

Laboratory findings were non-specific and did not suggest calciphylaxis. The patient's anaemia worsened and gradual acute-on-chronic renal failure was observed. Parathyroid hormone (PTH) levels were normal, as were calcium and phosphorous levels throughout the patient's admission. However, clinical suspicion of calciphylaxis was very high and a second biopsy was obtained. Histological findings suggested calciphylaxis almost 30 days after the patient was first admitted (Figs. 4 and 5).

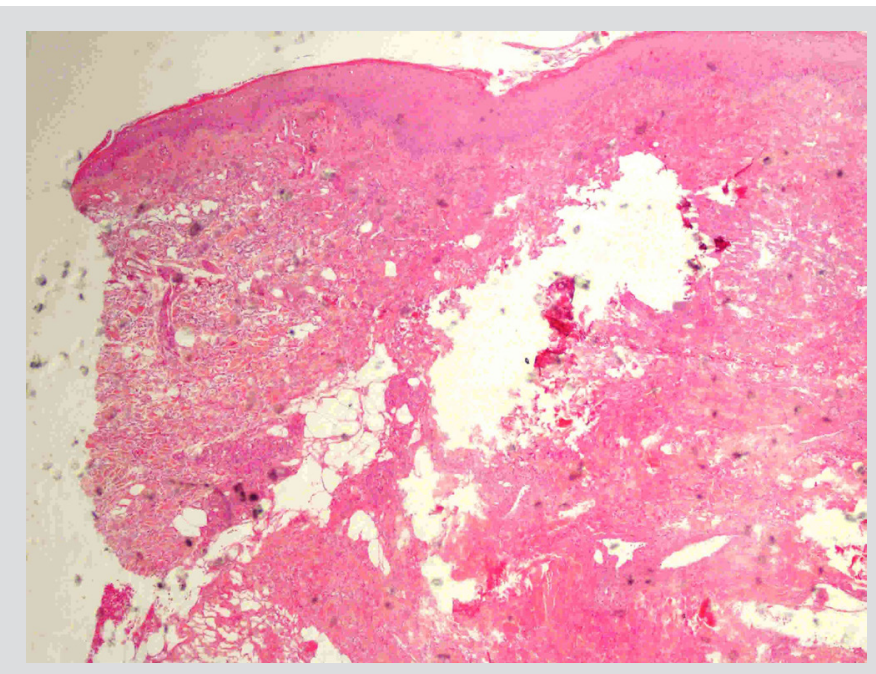

Figure 4. Skin biopsy histology (H\&E, 4x) showing necrosis and inflammation (centre)

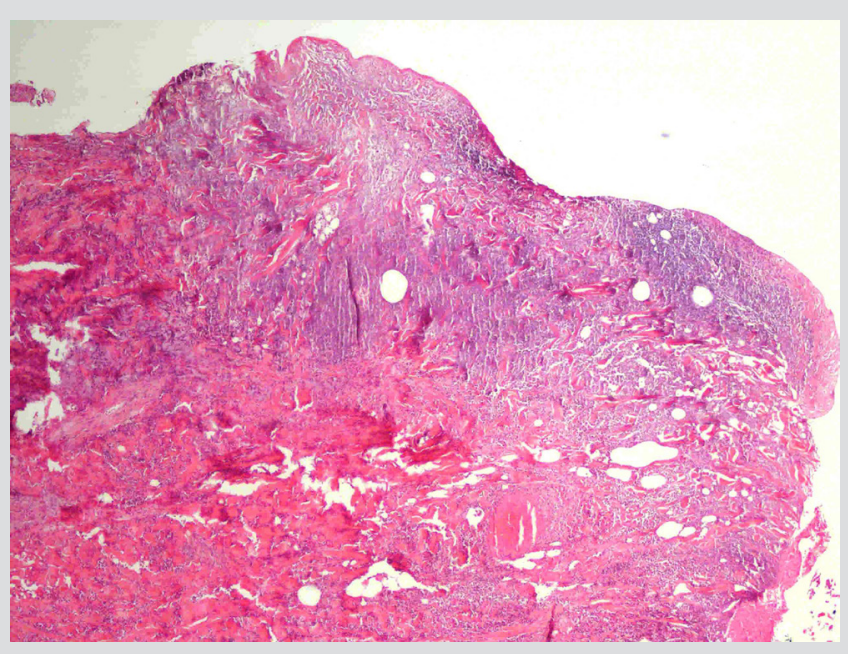

Figure 5. Skin biopsy histology (H\&E, 4x) showing ulceration, necrosis and calcification (left) 
During this time, general surgery clinicians were responsible for wound care and the patient underwent surgical and chemical debridement. Different types of dressings were applied but negative pressure wound therapy was unavailable. Nevertheless, superimposed infection developed despite this satisfactory care. For the first 10 days of her admission, the patient received empirical antibiotic therapy with ceftazidime, with treatment later escalated to ceftriaxone for 12 more days after the identification of Morganella morganii and Citrobacter freundii.

Although a clean wound bed was achieved after 2 weeks, new lesions developed and the wound again became infected. The patient was started on imipenem and vancomycin. Newly obtained blood cultures revealed methicillin-resistant Staphylococcus aureus, Klebsiella pneumoniae and Pseudomonas aeruginosa. A few days later the patient died from refractory septic shock and multi-organ failure.

\section{DISCUSSION}

In retrospect, the patient had many risk factors strongly associated with calciphylaxis: she was a woman aged over 60 with chronic kidney disease and a recent diagnosis of oligoarthritis ${ }^{[1]}$. However, she did not have other risk factors such as obesity, hypoalbuminemia, hyperphosphatemia, diabetes, long-term dialysis or hypercoagulable states, but had been on warfarin and calcium carbonate for over a decade, received regular iron supplementation and had recently been started on glucocorticoids, all of which are strongly associated with calciphylaxis ${ }^{[2]}$.

The patient presented with non-uremic calciphylaxis which is the least common form, the lesions were distal rather than proximal, there were no relevant changes in calcium-phosphorus metabolism and the first biopsy was not conclusive.

Systematic reviews have found a strong association between non-uremic calciphylaxis and warfarin, and also with glucocorticoid use and autoimmune disorders. These can manifest in patients with only mild chronic kidney disease, with creatinine levels as low as $1.2 \mathrm{mg} / \mathrm{dl}$ and even with an estimated glomerular filtration rate over $60 \mathrm{ml} / \mathrm{min} / 1.73 \mathrm{~m}^{2[3]}$. In our case, glucocorticoids had been introduced approximately 1 year before the lesions appeared, and may have been the final precipitating factor in a fragile patient on the verge of microvascular calcification.

Different mechanisms have been proposed for calciphylaxis: small vessel calcification resulting from chronic kidney disease, mineral bone disease, supplementation with calcium phosphate or vitamin D, and chronic inflammation. Deficient inhibition of vascular calcification is also important and is the mechanism by which vitamin $\mathrm{K}$ inhibitors like warfarin seem to contribute.

There are no guidelines on optimal treatment. Most publications focus on the need for multidisciplinary care, as supplied to this patient, who received satisfactory wound management and pain control. Some of the literature mentions the long-term off-label use of sodium thiosulfate, but this patient required rapid treatment as the lesions were progressing very quickly. Skin patches have also been shown to be effective, but only when ulcers stop spreading and skin and subcutaneous tissue is viable ${ }^{[4]}$.

The antibiotic therapy threshold should be low. The high mortality of up to $80 \%$ associated with this condition is due to superimposed infection and septic shock. Control of infection, pain management and wound care should be the main goals of treatment ${ }^{[5]}$.

Only by raising awareness and reporting such cases can we, as a medical community, foresee and recognize conditions like calciphylaxis, so that the next time the perfect storm is forming, we are able to chart the correct course.

\section{REFERENCES}

1. Nigwekar S, Thadhani R, Brandenburg V. Calciphylaxis. New Engl J Med 2018;379:399-400.

2. Brandenburg VM, Cozzolino M, Mazzaferro S. Calcific uremic arteriolopathy: a call for action. Semin Nephrol 2014;36:641-647.

3. El-Azhary RA, Patzelt MT, McBane RD, et al. Calciphylaxis: a disease of pannicular thrombosis. Mayo Clin Proc 2016;91:1395-1402.

4. McCarthy JT, El-Azhary RA, Patzelt MT, et al. Survival, risk factors, and effect of treatment in 101 patients with calciphylaxis. Mayo Clin Proc 2016;91:1384-1394.

5. Brandenburg VM, Kramann R, Rothe H, et al. Calcific uraemic arteriolopathy (calciphylaxis): data from a large nationwide registry. Nephrol Dial Transplant 2017;32:126-132. 